the area covered is urban with good access to a variety of health and social care services.

In 2010 the hospice were asked to provide a similar service providing advice on palliaitive care/specialist palliative care to health care professionals across all geographical areas of North Wales acting as a triage centre for palliative medicine consultant advice, this service was not made avaialble to patients and families due to concerns regarding potential volume of calls. This introduced a population located in large rural areas with more sporadic access to services.

Aim \& Approach The purpose of the audit was to encourage discussions about the ethics of delivering a two tier service based purely on geography but also considers the context of the calls in terms of the services available to patients within each locality in terms of model of service provided and also the hours of operation/delivery

Outcomes This presentation details the findings of an audit of the advice call forms completed reviews and compares the data collected from both geographical areas covered by the advice line, considering the volume of calls from both areas, the time and duration of calls and also the reasons why people called.

Application to practice The audit recognises some limitations in the method by which data is currently collected and reviewed providing guidance for the future in terms of what do we need to know in order to measure the impact and outcome of the provision of this service to patient $s$ and their families.

The work also draws conclusions for practice in terms of identifying current need within our locality.

\section{P10 HOSPICE RAPID RESPONSE SERVICE - A PROSPECTIVE ANALYSIS}

Beverley Clayton, Lesley Spencer. Princess Alice Hospice, Esher, UK

10.1136/bmjspcare-2013-000591.32

Background/context Following a retrospective analysis of urgent unplanned visits, evidence emerged that supported a new rapid response role for a clinical nurse specialist.

\section{Main Aims}

- To enable palliative care patients in the community, who have complex needs or whose condition is deteriorating, to access prompt specialist palliative care services.

- To assess whether more patients

- are enabled to exercise their preference about place of death

Approach used The service was available to new and existing patients for end of life care and support of urgent symptom control issues $(\mathrm{N}=78)$. Referrals requiring urgent attention were passed onto the Rapid Response Nurse. The CNS worked flexible hours in order to support the needs of the patient. Data was collected prospectively over a six month period using survey monkey.

Outcomes Seventy Eight patients supported over 6 month period. $38 \%$ had a non - malignant diagnosis. $67 \%$ of patients were referred for terminal care with the remaining requiring symptom control. As a result of the rapid response visit $86 \%$ of patients remained in their own home. None were transferred into the acute sector. $75 \%$ of patients who died within the 6 month period achieved their preferred place of death with only $6 \%$ who wished for a home death unable to achieve this.
Application to hospice practice The service was timely, responsive and supported increasing numbers of patents to be cared for and to die in their preferred place of death. All visits responded to crisis therefore all patients had potential to be admitted to hospital. Patients in the Nursing homes were particularly vulnerable.

The service therefore prevented unnecessary admissions into the acute sector and supported Hospital Palliative Care Teams with rapid discharge home.

A Rapid Response Clinical Nurse specialist is effective - right skill, right intervention, right time.

\section{P11 THE STARS THAT COME OUT AT NIGHT - PRESENTING THE HOSPICE '8 TO 8' NIGHT NURSING SERVICE}

Dawn Neil-Jones, Lesley Spencer. Princess Alice Hospice, Esher, United Kingdom

\subsection{6/bmjspcare-2013-000591.33}

Background/context Maximum impact in terms of achieving patients preferred place of care may be gained by focussing future service development on extending and improving care at home. This also ensures on-going collaboration between NHS and other providers of end of life care.

Since September 2011 the Hospice has been developing the ' 8 to 8 ' service which provides a night nursing service for existing Hospice patients and more recently, through partnership working with other healthcare providers for dying patients not known to the Hospice.

Main Aims

- To optimise the opportunity for patients requiring end of life care who wish to stay at home to remain in their preferred place of care

- To prevent unnecessary admissions to hospitals

Approach used The service is delivered by both bank and permanent members of staff and includes both registered nurses and senior health care assistants. All staff are inducted by the Hospice and given the requisite palliative care skills. The service is led by a clinical nurse specialist who supports and co-ordinates the allocation of staff according to clinical need. The nurses remain with the patient throughout the night.

Outcomes Evaluation demonstrates that the service has exceeded expectations. Most patients referred to the 8 to 8 service are for terminal care with $95 \%$ of patients achieving their preferred place of death. The service now delivers 50 nights per month across the catchment area.

Application to hospice practice The 8 to 8 service has been commissioned by two clinical commissioning groups since its inception and continues to expand and build on its success. It is delivered by specially trained nurses who are compassionate, efficient, caring and greatly improve the patient experience. Increasing numbers of complex patients are now able to be cared for in their preferred place of care.

\section{P12 HOSPICE AT HOME SERVICE LISTENING, RESPONDING, DEVELOPING}

Vanessa Gibson, Liza McEvilly, Robert Standfield. St Richards Hospice, Worcester, UK

\subsection{6/bmjspcare-2013-000591.34}

In 2008 the Department of Health (DH) identified increasing evidence that lack of co-ordinated community support was 